\title{
RECHERCHES SUR LA PIROPLASMOSE DU PORC
}

\section{EN BULGARIE}

\author{
Par P. PAVLOV et II. PASCHEV
}

L'étude sur la piroplasmose du porc, publiée par Cèrruti en 1939, a attiré notre attention et, comme les facteurs géo-climatiques sont semblables en Italie et dans les Pays balkaniques, l'existence de la maladie nous parut probable en Bulgarie ; l'éclaircissement de cette question, importante au point de vue économique, a fait l'objet des recherches que nous allons exposer.

Historique. - La maladíe fut constatée pour la première fois en U.R.S.S. par Dementiev et Kovalevski (1911), chez des pores malades dont les globules rouges présentaient des formations arrondies et annulaires qui auraient disparu après traitement par la quinine et l'atoxyl. Trautmann (1914) a retrouvé la maladie en Afrique orientale allemande et décril le parasite que Knuth et Du Toit (1921) nommèrent Piroplasma trautmanni et dont ils montrèrent les affinités morphologiques avec $P$. bigeminum, $P$. canis et $P$. caballi, Dans ces parasites, Trautmann nota un dimorphisme de l'appareil nucléaire : très souvent, un grand noyau d'aspect làche, et, plus rarement, un noyau petit, rond et compact.

Sparapani (1917) a signalé trois cas de piroplasmose porcine à l'abattoir de Venise, chez des animaux àgés de 9 à 11 mois, qui avaient montré des signes cliniques et des lésions typiques de la maladie. L'examen du sang avait révélé la présence, dans les globules rouges, de parasites que l'auteur identifia à tort avec Piroplasma ovis. Lanzillo (1924), en collaboration avec Fusco et Scandaliato, constata l'existence de la maladie dans la région de Grosseto ; en 1933, il en donna une étude plus complète. D'après lui, elle frapperait surtout les races sélectionnées et, seulement exceptionnellement, les races locales ; elle sévit surtout au printemps et à l'automne, plus rarement aux autres saisons. Lanzillo a déerit deux formes cliniques de la maladie, l'une maligne, à type septicémique, avec température de 41 à $42^{\circ}$ C., l'autre bénigne, chronique, presque toujours sans hémoglobinurie; la première donne surtout des lésions de seplicémie, la seconde, une anémie grave.

ANn. de PAnasitologie, T. XXI, Nos 5-6, 1946, p. 235-240. 
Cerruti (1934) a signalé la maladie en Sardaigne, dans la province de Cagliari.

Aboussalimov (1935) a décrit en U.R.S.S. deux cas avec signes cliniques et lésions typiques de la maladie. L'examen du sang lui montra dans un cas Piroplasma trautmanni et, dans l'autre, des parasites arrondis et près de deux fois plus petits.

Cerruti, en 1939, publia un important mémoire sur la piroplasmose du porc en Italie, dans lequel, à côté du P. trautmanni, il décrivit 'un autre parasile, qu'il nomma Babesiella perroncitoi; il signala aussi l'exislence de corps anaplasmoïdes. Ses recherches ont jeté, une vive lumière sur cette question. Ce qui, toutefois, est le moins élucidé à ce sujet et qui doit encore faire l'objet de recherches spéciales, c'est la question des hòtes intermédiaires.

En Bulgarie, le problème de la piroplasmose du porc était entièrement nouveau. En 1943, Yanev signala deux cas d'affections dues à $P$. trautmanni, constatées sur des frottis de sang envoyés, I'un de Panaghourichtsche et l'autre de Burgas. Après cette constatalion, faite à Stara-Zagora, Pavlov, dont les recherches jusqu'alors étaient restées négatives, attira à nouveau l'attention sur la question et donna des instructions spéciales pour la recherche de la maladie. Le résultat fut la découverte de 19 cas constatés : 16 à Burgas, 2 à Vesselia et 1 à Dolno-Esserovo.

Mais nous pensons que la maladie existe aussi dans d'autres régions, et spécialement en Bulgarie du Sud; même dans la région de Burgas, des foyers doivent exister, qui ne sont pas encore connus, En outre, la maladie, telle qu'on l'a constatée jusqu'ici en Bulgarie, se manifeste sous une forme bénigne, accompagnée de symptômes discrets, ce qui ne facilite pas son dépistage.

Symptômes cliniques. - Sparapani (1917) écrit que les pores malades présentent des muqueuses sub-ictériques, une démarche chancelante et un amaigrissement considérable. Lanzillo (1933) décrit deux formes de la maladie : une forme maligne, à type septicémique avec fièvre à $41-42^{\circ} \mathrm{C}$., dyspnée, anorexie, démarche chancelante, muqueuses hyperémiées et hémoglobinurie, et, d'autre part, une forme bénigne chronique à type d'anémie et presque toujours sans hémoglobinurie. Aboussalimoy (1935) décrit comme symptômes une température à $40-41^{\circ}$ C., des muqueuses ictériques et pâles, de la faiblesse, des troubles de la démarche, de l'anorexie avec constipation ou diarrhée. Cavallelti (1938) décrit des signes analogues à ceux des auteurs précédents. Cerruti (1939), dans sa description détaillée, reconnait une forme typique qu’il appelle 
classique et une autre plus bénigne à symptomatologie plus discrète, se manifestant presqu'exclusivement par une anémie, avec température de $39,9-40^{\circ}$ C., des muqueuses porcelaine ou sub-ictériques et des troubles gastro-intestinaux.

Les constatations faites en Bulgarie montrent une symptomatologie identique à celle de la « forme bénigne chronique » de Lanzillo et de la « forme bénigne, » de Cerruti. C'est vraisemblablement pour cela qué la maladie y avait jusqu'alors échappé à l'attention.

Les symptômes que nous avons observés sont les suivants : affaiblissement, perte de l'appétit; les animaux perdent leur vivacité habituelle et restent couchés sur le sol ; excitation nerveuse avec tremblements; démarche chancelante. Il s'y ajoute des signes digestifs, caractérisés par une constipation suivie de diarrhée; la défécation s'accompagne de ténesme; les muqueuses sont pâles avec une teinte porcelaine; il existe une anémie plus ou moins prononcée, avec une température allant de 39 à $42^{\circ}$ C., oscillant par accès ; l'urine est normale ou concentrée avec une teinte de cognac. On n'a pas constaté d'ictère, d'œedème des conjonctives, ni d'hémorragies. Avant la mort, la température tombe brusquement à 37,2$38^{\circ} \mathrm{C}$., avec un pouls de 80 à 130 et une respiration de 60 à la minute. Dans le sang, on constate une mono-, thrombo- et lymphocytose ; il y a diminution des polynucléaires neutrophiles.

Anatomie pathologique. - Les lésions macroscopiques et microscopiques ont été décrites en détail par Cerruti. Nous n'avons pu malheureusement faire de recherches sur ce point, tous les malades ayant été traités et guéris, et les deux seuls pores, morts de la maladie, n'ayant pas été mis à notre disposition. Trois autres animaux, sacrifiés au début de la mảadie, ne nous ont montré que des hémorragies gastro-intestinales et vésicales et une très faible splénomégalie.

Agent étiologique. - Les frottis de sang nous ont montré un parasitisme faible des globules rouges par des- piroplasmes, diagnostiqués comme $P$. trautmanni.

Les formes que nous avons observées sont variables. Les plus typiques sont les éléments' piriformes, puis des éléments ronds, annulaires, ovales ou plasmodiens, contenant un noyau marginal ou central arrondi, avec chromatine colorée en rouge vif. Les formes ovales ont de $2 \mu, 3$ à $3 \mu, 4$ sur $1 \mu, 1$ à $2 \mu, 4$. Le diamètre des formes rondes varie de $1 \mu, 6$ à $4 \mu$. Les éléments piriformes mesurent de $2 \mu$ à $6 \mu$ sur $0 \mu, 8$ à $2 \mu, 4$. Les parasites que nous avons vus ne s'écartent pas de la morphologie elassiquement connue. Une 
hématie en contient habituellement un ou deux ; s'il s'agit d'éléments piriformes, ils sont alors disposés à angle aigu (fig. 1). Le pourcentage d'invasion des globules rouges varie de 0,5 à 1 p. 100 .

Dans un frottis du sang d'un malade, nous avons vu, en plus des piroplasmes, deux corps indéterminés, paraissant de nature parasitaire (fig. 2). Il s'agit de deux éléments extra-cellulaires de $4 \mu, 8$ sur $1 \mu, 2$ ressemblant à des trypanosomes sans flagelles ou plutôt aux sporozoaires du Soudan, décrits par Archibald et Susu (1924), mais avec un protoplasme en réseau et un petit noyau excentrique, contenant un grain de chromatine compact, coloré en rouge par le Giemsa. Nous ne pouyons dire s'il s'agit d'un parasite ou d'une souillure de la préparation.

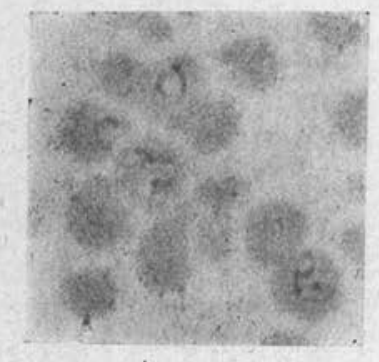

FIG. 1

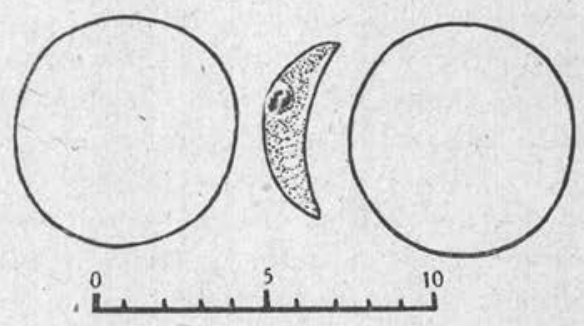

FIG. 2

Agent vecteur. - On pense que la maladie serait transmise, en Afrique du moins, par la tique Boophilus decoloratus. Cerruti a trouvé sur les pores malades les tiques suivantes: Rhipicephalus sanguineus, Hyalomma rgyptium et Dermacentor reticulatus.

Pour notre part, et malgré toutes nos recherches, nous n'avons pas rencontré de tiques sur les animaux malades; il est à remarquer que tous ceux-ci vivaient au voisinage des basses-cours; cela ne veut pas, toutefois, dire que ce soient les Argasinés qui sont les vecteurs de l'infection.

Epidémiologie. - La maladie existe en quantité non négligeable dans le pays et spécialement dans la région de Burgas où, comme nous l'avons dit, il est probable que tous les foyers ne sont pas encore découverts. Il est intéressant de constater qu'elle existe chez les pores d'une ville (Burgas), qui sont élevés dans des conditions modernes et ne vont pas au pâturage ou en forêt. Jusqu'à présent, 19 cas ont été constatés. Leur répartition saisonnière est indiquée dans le tableau ci-dessous : 
Avril .................

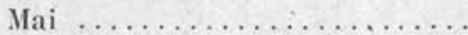

Juin $\ldots \ldots \ldots \ldots \ldots \ldots \ldots \ldots \ldots$

Juillet

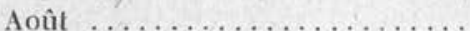

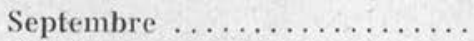

Octobre

Avril-Octobre 1945

$\begin{array}{rr}- & - \\ 2 & 2 \\ 3 & - \\ 3 & 2 \\ 7 & 1 \\ 9 & 4 \\ 9 & 5 \\ - & 5 \\ 33 & -\end{array}$

-
-
1
2
3
4
4
-14

Cela confirme la constatation de Lanzillo que la piroplasmose du porc est surtout une maladie d'automne.

Le nombre des cas est encore trop limité pour en déduire le ròle réel de la race et de l'âge des animaux; tous étaient àgés de 5 à 10 mois et de race sélectionnée. Il sera intéressant de rechercher la maladie parmi les pores de la région de Sredez, près de Burgas, qui sont de race locale et vivent à l'état demi-sauvage dans les forêts.

Thérapeutique. - Dementiev (1911) a obtenu la disparition des parasites par injections de quinine et d'atoxyl. L'A pirosome a donné de bons résultats à Lanzillo (1933), l'A caprin à Cerruti (1939).

Nous avons employé aussi l'Acaprin avec d'excellents résultats ; nous avons utilisé des doses de 0,5 à 1 ce. d'une solution à 5 p. 100 ou de 5 à $10 \mathrm{cc}$. d'une solution à 5 p. 1.000. Le médicament est mieux supporté après administration préalable de Cardiazol. Tous. nos animaux avaient été traités un à deux jours après apparition des signes cliniques et établissement du diagnostic; ils ont tous guéri.

\section{RÉsumé}

Les auteurs signalent l'existence de la piroplasmose porcine en Bulgarie, dans la région de Burgas, et surtout chez des porcs provenant de la ville de Burgas. La maladie a été constatée chez des pores àgés de 5 à 10 mois et de race sélectionnée. Elle est due à Babesia trautmanni. Cliniquement, elle se présente sous l'aspect de la forme bénigne. Il n'a pas été trouvé de tiques sur les malades ; la maladie est à prédominance automnale. Le traitement par l'Acaprin a donné de bons résultats dans tous les cas. Deux corps extracellulaires, de nature indéterminée, ont été rencontrés dans les frottis de sang d'un malade. 


\section{BIBLIOGRAPHIE:}

Aboussalimov (M.). - Sovietskaia Veterinarna, n 6, p. 60, 1935.

Brumpt (E.). - Précis de Parasitologie, 5 édit., Paris, 1934.

Cavalletti (S.). - Nuova Veterinaria, XVI, p. 114, 1938.

Cennuti (C.-G.). - Annales de Parasitologie, XVII, p. 114, 1939,

Curasson (G.). - Traité de Protozoologie vétérinaire, III, Paris, 1934.

Нутүва el Мавек, - Spezielle Path. u. Therapie d. Haustiere, 1, 8* éd., 1941.

Jacor (E.). - Parasitische Protozoen in Mensch. u. Tier., Berlin, 1941.

KNuth u. Du Tort. - In Mense's Hbch Tropenkrheit., VI, 1921.

Meteloin, Markov el Kazanski. - In Parasitol. Vétérin. sous la rédaction de Skriabine, I à II, Moscou, 1939.

Neveu-Lemaine, - Traité de Protozoologie Méd. et Vét., Paris, 1943.

Pavlov (P.-C.). - Dtsch. Tropmedizin. Ztschr., 48, p. 25, 1944.

Veterinarna Sbirka, XLVIII, p. 18, 1945.

WENYON (C.-M.). - Protozoology, London, 1926.

YANEv (E.-L.). - Belgarska Veterinarna Kliniska, Bd. I, Ht. 9/10, S. 3, 1943.

YакIMOV (W.-L.). - Bol. D.

Service antiparasitaire de l'Institut central vétérinaire bactériologique de l'Etat (Sofia) et Station vétérinaire bactériologique de l'Etat (Burgas) 